\title{
Fascin-1 depletion from hepatocellular carcinoma cells inhibits migfilin and vasodilator-stimulated phosphoprotein expression and enhances adhesiona
}

\author{
Vasiliki Gkretsi ${ }^{1}$, Dimitrios P. Bogdanos ${ }^{1,2,3}$
}

${ }^{1}$ Department of Biomedical Research and Technology, Institute for Research and Technology-Thessaly, Centre for Research and Technology-Hellas (CE.R.T.H.), 41222 Larissa, Greece

${ }^{2}$ Department of Rheumatology, School of Medicine, University Hospital of Larissa, University of Thessaly, 41110 Larissa, Greece ${ }^{3}$ Department of Liver Studies and Transplantation, Institute of Liver Studies, King's College Hospital, Denmark Hill, London SE5 $9 R S, U K$

\begin{abstract}
Aim: Extracellular matrix (ECM)-adhesions and their interaction with actin cytoskeleton are fundamental for hepatocellular carcinoma (HCC). Fascin-1, an actin-bundling protein, is correlated with poor HCC prognosis, and is known regarding the molecular mechanism of its action. In this study, the authors investigated Fascin-1 basic molecular mechanism and cellular properties in HCC cells. Methods: Fascin-1 was silenced by small interfering RNA and the expression of actin. The ECM-adhesion-related proteins were assessed along with the cells' adhesion capacity in two cell lines that differ in terms of aggressiveness; the hepatoma cell line PLC/PRF/5 (Alexander) and the highly invasive HCC cell line HepG2. Results: This study shows that Fascin-1 is upregulated in HepG2 cells compared to Alexander cells and when silenced leads to increased cell adhesion only in HepG2, while at the same time is associated with reduced migfilin and vasodilator-stimulated phosphoprotein (VASP) expression. Conclusion: This is the first study to show that Fascin-1 contributes to a more aggressive phenotype in HCC cells and acts through migfilin and VASP.
\end{abstract}

Key words: Adhesion; Fascin-1; hepatocellular carcinoma; migfilin; vasodilator-stimulated phosphoprotein

\section{Address for correspondence:}

Dr. Vasiliki Gkretsi, Department of Biomedical Research and Technology, Institute for Research and Technology-Thessaly, Centre for Research and Technology-Hellas (CE.R.T.H.), 51 Papanastasiou Street, 41222 Larissa, Greece. E-mail: vasso.gkretsi@gmail.com

Received: 23-05-2015, Accepted: 09-10-2015

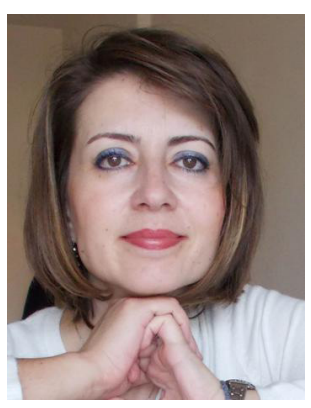

Dr. Vasiliki Gkretsi received her BSc in Biology from the University of Athens, Greece (2001) and her Ph.D from the University of Pittsburgh Medical School, USA (2006). She has received numerous awards and published 21 papers (h index $=12$ ). Her research focuses on the role of extracellular matrix, and cell adhesion in metastasis.

\begin{tabular}{|l|c|}
\hline \multicolumn{3}{|c|}{ Access this article online } \\
\hline \multirow{2}{*}{$\begin{array}{l}\text { Website: } \\
\text { http://www.hrjournal.net/ }\end{array}$} \\
\hline
\end{tabular}

This is an open access article distributed under the terms of the Creative Commons Attribution-NonCommercial-ShareAlike 3.0 License, which allows others to remix, tweak, and build upon the work non-commercially, as long as the author is credited and the new creations are licensed under the identical terms.

For reprints contact: service@oaepublish.com

How to cite this article: Gkretsi V, Bogdanos DP. Fascin-1 depletion from hepatocellular carcinoma cells inhibits migfi lin and vasodilatorstimulated phosphoprotein expression and enhances adhesion. Hepatoma Res 2016;2:42-6. 


\section{INTRODUCTION}

Extracellular matrix (ECM), focal adhesions, and their interaction with actin cytoskeleton are fundamental for a number of vital cellular processes such as cell survival, differentiation, development, and tissue homeostasis. This is particularly true for the organ liver as the main parenchymal cells of the liver, hepatocytes, interact to a great extent with ECM, and express a number of focal adhesions and actinrelated proteins. Moreover, the fact that ECM and ECMrelated proteins are fundamental for liver biology is evident by a number of studies showing how they affect hepatocyte differentiation, ${ }^{[1]}$ survival, ${ }^{[2]}$ and normal liver function. ${ }^{[3,4]}$ More specifically, matrix overlay on primary hepatocytes cultured in vitro inhibits the dedifferentiation that normally occurs due to culturing and maintains hepatocytes in a fully differentiated state. ${ }^{[5]}$ Along the same line, loss of a cell adhesion protein related to integrins (namely, integrin-linked kinase) from mouse hepatocytes in vitro and in vivo leads to increased apoptosis and hepatitis, ${ }^{[2]}$ showing clearly that cell-ECM interactions are critical for hepatocyte survival. Finally, liverspecific ablation of integrin-linked kinase leads to abnormal liver histology ${ }^{[4]}$ and increased liver regeneration capacity, ${ }^{[3]}$ indicating the crucial role played by cell-ECM adhesion proteins in liver structure and function.

Moreover, ECM plays central role during carcinogenesis in the liver, as hepatocellular carcinoma (HCC) is known to result from a series of events which include fibrosis, cirrhosis, generation of phenotypically-altered hepatocytes, and dysplasia ultimately leading to HCC. ${ }^{[6]}$ Thus, cell adhesion proteins that connect cells to the ECM and/or to neighboring cells are evidently fundamental for HCC development and progression. Moreover, cell adhesion proteins are often directly or indirectly connected to actin cytoskeleton creating a network of interacting proteins that is crucial for tissue homeostasis. Interestingly, most of these proteins are found to be deregulated in cancer and cancer metastasis. ${ }^{[7,8]}$ This deregulation destabilizes cell attachment to the surrounding ECM and neighboring cells, thus facilitating cancer cells dissociation from the original tumor mass and invasion of surrounding tissues, and ultimately leads to metastases formation.

Fascin- 1 is an actin-bundling protein that is found in membrane ruffles and stress fibers, and its expression has been shown to be greatly increased in many transformed cells ${ }^{[9]}$ and a spectrum of cancers such as breast cancer, colon cancer, pancreatic cancer and prostate cancer. In HCC in particular, Fascin-1 has been correlated with poor prognosis..$^{[10]}$

Moreover, Fascin-1 has been suggested as a novel marker of HCC progression and a significant indicator of poor prognosis for HCC patients. ${ }^{[1]}$ However, little is known regarding the molecular mechanism of its action. In a recent work, we have shown that Fascin-1 is negatively regulated by migfilin, ${ }^{[12]}$ a novel cell-matrix adhesion protein known to interact with vasodilator-stimulated phosphoprotein (VASP), ${ }^{[13]}$ and is localized both at cell-matrix and cell-cell adhesions. ${ }^{[14]}$

In the present study, we tested the expression and molecular mechanism of the action of Fascin- 1 in two liver cell lines that differ in terms of aggressiveness; the hepatoma cell line PLC/PRF/5 (Alexander) and the highly invasive HCC cell line HepG2.

\section{METHODS}

\section{Liver cell lines}

Two liver cell lines of different invasive capacity were used in the present study; the hepatoma cell line PLC/PRF/5 (Alexander) and HCC cell line HepG2. Both cell lines were purchased from American Type Culture Collection.

\section{Transfection with small interfering RNAs}

Both Alexander and HepG2 cells were treated for $48 \mathrm{~h}$ with $100 \mathrm{nmol} / \mathrm{L}$ small interfering RNA (siRNA), non-specific control (NSC) siRNA, or siRNA against Fascin-1 using the Lipofectamine 2,000 transfection reagent (Invitrogen, Carlsbad, CA, USA) according to the company's guidelines. The siRNA sequence used to silence Fascin was as purchased from Santa Cruz, while the sequence 5'AAA CUC UAU CUG CAC GCU GAC3' was used as NSC. Silencing efficiency prior to every experiment performed was tested by western blot.

\section{Antibodies}

Anti- $\beta$-actin antibody (Sigma-Aldrich) was used as loading control. Antibodies VASP and Fascin-1 were purchased from Cell Signaling. The monoclonal antibody against migfilin was kindly provided by Dr. Chuanyue Wu (Professor at the University of Pittsburgh Medical School, Pittsburgh, PA, USA).

\section{Protein extraction and western blot analysis}

Total cell lysates were obtained using $1 \%$ sodium dodecyl sulfate in radioimmunoprecipitation assay buffer $(20 \mathrm{mmol} / \mathrm{L}$ Tris/Cl pH 7.5, 150 mmol/L NaCl, 0.5\% NP-40, 1\% TX-100, $0.25 \%$ sodium deoxycholate, $0.6-2 \mu \mathrm{g} / \mathrm{mL}$ aprotinin, $10 \mu \mathrm{mol} / \mathrm{L}$ leupeptin, and $1 \mu \mathrm{mol} / \mathrm{L}$ pepstatin). Protein concentrations in the samples were determined by the BCA protein assay kit (Pierce) using bovine serum albumin as standard. An equal amount of protein was loaded on each lane of a 10-12\% acrylamide gel and transferred to a PVDF membrane (Millipore) using the Bio-Rad Semi-dry transfer system (Bio-Rad, Hercules, CA, USA). Signals were detected using suitable secondary immunoglobulin $\mathrm{G}$, conjugated with horseradish peroxidase (Invitrogen). Antibody detection was performed using super-signal ECL detection system (Pierce).

\section{Cell adhesion assay}

Cell adhesion assay was performed as described previously. ${ }^{[13]}$ Briefly, cells were transfected with a control NSC siRNA or siRNA against Fascin-1. Forty-eight hours post-transfection, $10^{4}$ cells/ well were seeded in 6 wells of a 96-well plate precoated with $0.1 \%$ gelatin. After a 60 -min incubation at $37^{\circ} \mathrm{C}$, 
three of the wells were washed three times with phosphatebuffered saline while the remaining three were fixed with 4\% paraformaldehyde (PFA). Washed wells were also fixed with PFA and then cells in all wells were quantified using crystal violet. ${ }^{[13]}$ Crystal violet was washed using $\mathrm{ddH}_{2} \mathrm{O}$ and cells were solubilized using acetic acid. Absorbance was measured at $570 \mathrm{~nm}$ using Perkin Elmer EnSpire plate reader (Waltham, MA, USA). Adhesion was presented as the ratio of the absorbance at $570 \mathrm{~nm}$ of adhered cells (washed) divided by the absorbance at $570 \mathrm{~nm}$ of the total seeded cells (not washed). The data from two independent experiments were analyzed using the Student's $t$-test. $P<0.05$ was considered statistically significant.

\section{Statistical analysis}

Comparison of means using Statgraphics sof tware (Statgraphics Company, Warrenton, VA, USA) was used for the statistical analysis. $t$-test was performed, and $P<0.05$ was considered statistically significant.

\section{RESULTS}

\section{Fascin-1 protein expression is dramatically elevated in} HepG2 compared to Alexander cells

We first tested Fascin-1 protein expression in Alexander and HepG2 cells using western blotting. As shown in Figure 1a, Fascin-1 protein expression was found to be dramatically elevated in the highly invasive HepG2 cells compared to the

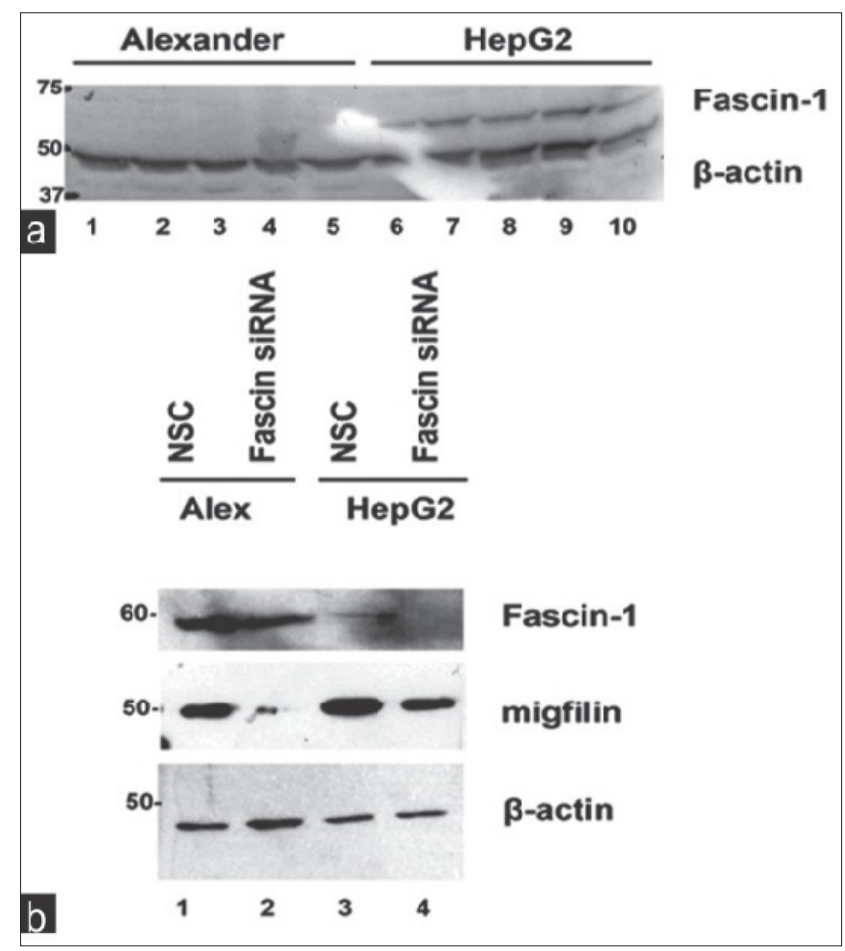

Figure 1: Fascin-1 is upregulated in HepG2 cells compared to Alexander while its depletion leads to a reduction in migfilin expression. (a) Representative western blot showing Fascin-1 protein expression in the two hepatocellular carcinoma cell lines tested; the low invasiveness Alexander and the highly invasive HepG2 cells; (b) the effect of Fascin-1 silencing on migfilin protein expression. $\beta$-actin is used as loading control. NSC: nonspecific control nonaggressive Alexander cells [Figure 1a]. This piece of data further confirms a critical role played by Fascin-1 in HCC and cancer cell aggressiveness.

\section{Fascin-1 gene silencing leads to downregulation of both migfilin and VASP}

We then proceeded with knocking down Fascin-1 gene in both HCC cell lines to better understand its function as well as its effect on known ECM-related proteins. As shown in Figure 1b, Fascin-1 was successfully silenced in both cell lines transfected with Fascin-1 siRNA compared to the cells transfected with an NSC siRNA (compare lanes 2 and 4 with lanes 1 and 3).

As ECM and actin cytoskeleton are fundamental for HCC progression and aggressiveness, we tested the expression of focal adhesion proteins migfilin (also known as Filamin Binding LIM-protein-1) a novel LIM domain-containing protein present both at cell-ECM, ${ }^{[15]}$ cell-cell adhesions, ${ }^{[14]}$ and VASP, a focal adhesion phosphoprotein known to regulate actin polymerization. ${ }^{[16-18]}$ Interestingly, migfilin and VASP interact with each other and are implicated in cellular adhesion to ECM as well as migration. ${ }^{[13]}$

As shown in Figure 1b, migfilin was significantly downregulated upon Fascin-1 silencing indicating a connection between the two molecules. Interestingly, in addition to migfilin, VASP was also found to be downregulated [Figure 2a] following Fascin-1 knock-down, engaging both proteins in Fascin-mediated effects.

\section{Fascin-1 silencing leads to increased cell adhesion in HepG2 cells}

Since both migfilin and VASP played significant roles in cell adhesion, we next investigated whether Fascin-1 silencing affected the property of cells to adhere to ECM. Thus, we performed a series of adhesion assays on $1 \%$ gelatin in both cell lines using cells that were transfected with NSC or Fascin-1 siRNA. As shown in Figure 2b, inhibition of Fascin-1 expression by siRNA induces an increase in cell adhesion ability of HepG2 cells, whereas this is not the case for the less

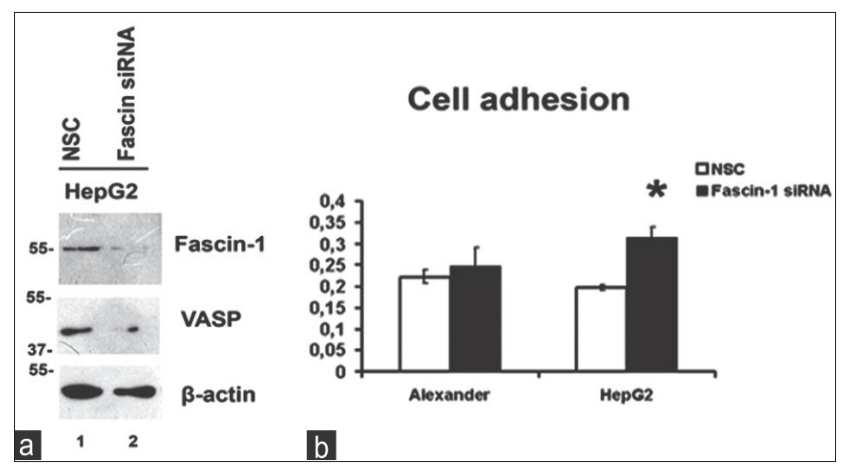

Figure 2: Fascin-1 silencing leads to VASP downregulation and promotion of cell adhesion. (a) The effect of Fascin-1 silencing in HepG2 cells on VASP protein expression assessed by western blotting. $\beta$-actin is used as loading control; (b) cell adhesion on $1 \%$ gelatin-coated 96 -well plates following Fascin-1 silencing in both cell lines. VASP: vasodilator-stimulated phosphoprotein; NSC: non-specific control 
invasive Alexander cells, which remain unaffected.

\section{DISCUSSION}

Cell adhesion proteins connecting cells to the ECM and/ or to the neighboring cells are often interconnected to the actin-cytoskeleton and this network of interacting proteins is fundamental for tissue homeostasis while at the same time being deregulated in cancer and cancer metastasis..$^{[7,8]}$ Fascin-1 is an actin-bundling protein that is found in membrane ruffles and stress fibers. ${ }^{[19]}$ The expression of Fascin-1 is greatly increased in many transformed cells, as well as in specialized normal cells including neuronal cells and antigenpresenting dendritic cells. A morphological characteristic common to these cells expressing high levels of Fascin- 1 is the development of many membrane protrusions in which Fascin-1 is predominantly present. ${ }^{19]}$ Recent studies show that Fascin-1 also localizes to invadopodia, membrane protrusions formed at the adherent cell surface that facilitate ECM invasion, thus providing a potential molecular mechanism for how Fascin-1 increases the invasiveness of cancer cells since Fascin-1 expression is upregulated in a spectrum of cancers such as breast cancer, colon cancer, pancreatic cancer, and prostate cancer. ${ }^{[20,21]}$ In HCC, in particular, Fascin-1 has been correlated with poor prognosis. ${ }^{\mid 10]}$

In fact, Fascin- 1 has been recently introduced as a migration factor associated with epithelial to mesenchymal transition in HCC cells facilitating their invasiveness in combination with matrix metalloproteinases. ${ }^{[2]}$ Moreover, it has been suggested to be a novel marker of progression in HCC and a significant indicator of poor prognosis for HCC patients. ${ }^{[1]}$ However, little is known regarding the molecular mechanism of its action.

In this study, we tested the expression and molecular mechanism of action of Fascin-1 in two HCC cell lines that differ in terms of aggressiveness; the hepatoma cell line PLC/PRF/5 (Alexander) and the highly invasive HCC cell line HepG2. Interestingly, we show that Fascin-1 is dramatically upregulated in HepG2 cells compared to more benign Alexander cells [Figure 1a].

We then utilized a siRNA-mediated silencing approach to knock-down the Fascin-1 gene. Fascin-1 silencing led to a reduction in the expression level of two important focal adhesion proteins related to the cytoskeleton, namely, migfilin $^{[14,15]}$ [Figure 1b], and its interactor VASP [Figure 2a]. ${ }^{[13]}$

More importantly, Fascin-1 silencing led to significantly increased cell adhesion in the highly invasive and aggressive HepG2 cells [Figure 2b] but had no effect on the less invasive Alexander cells, indicating that Fascin-1 silencing has, indeed, a great impact on more aggressive cells. Furthermore, the fact that it results in elevated cell adhesion in HepG2 cells shows that Fascin-1 depletion stabilizes cell-ECM adhesions leading to a less aggressive cancer phenotype. These findings are evidence confirming previous studies showing the potential of Fascin-1 as a therapeutic target for metastasis..$^{[9]}$
The fact that Fascin-1 silencing leads to migfilin and VASP downregulation and increased adhesion indicates that Fascin-1 may regulate migfilin and VASP and/or be physically associated with them. This evidence further complements recent work from our laboratory showing that migfilin silencing, among other things, reduces VASP expression, and leads to Fascin-1 upregulation, and promotion of cell adhesion in HepG2 cells. ${ }^{[12]}$ Therefore, the evidence clearly indicates a molecular interplay between the three proteins, migfilin, Fascin-1, and VASP, and the potential existence of a regulatory feedback loop in HCC cells.

Although our study was performed in cancer cell lines, which have their limitations in terms of modeling the physiological complexity of human cancer, it still offers significant insight into the molecular mechanism by which Fascin-1 is implicated in HCC pathogenesis. Of course, more studies are needed to decipher the exact sequence of molecular events taking place and the importance for HCC progression.

\section{Acknowledgments}

We are grateful to Dr. Chuanyue Wu, Professor at the University of Pittsburgh Medical School, Pittsburgh, PA, USA for providing us with the anti-migfilin monoclonal antibody.

\section{Financial support and sponsorship}

This study was supported by the European Association for the Study of the Liver Sheila Sherlock fellowship 2012.

\section{Conflicts of interest}

There are no conflicts of interest.

\section{REFERENCES}

1. Runge D, Runge DM, Bowen WC, Locker J, Michalopoulos GK. Matrix induced re-differentiation of cultured rat hepatocytes and changes of CCAAT/enhancer binding proteins. Biol Chem 1997;378:873-81.

2. Gkretsi V, Mars WM, Bowen WC, Barua L, Yang Y, Guo L, St-Arnaud R, Dedhar S, Wu C, Michalopoulos GK. Loss of integrin linked kinase from mouse hepatocytes in vitro and in vivo results in apoptosis and hepatitis. Hepatology 2007;45:1025-34.

3. Apte U, Gkretsi V, Bowen WC, Mars WM, Luo JH, Donthamsetty S, Orr A, Monga SP, Wu C, Michalopoulos GK. Enhanced liver regeneration following changes induced by hepatocyte-specific genetic ablation of integrin-linked kinase. Hepatology 2009;50:844-51.

4. Gkretsi V, Apte U, Mars WM, Bowen WC, Luo JH, Yang Y, Yu YP, Orr A, St-Arnaud R, Dedhar S, Kaestner KH, Wu C, Michalopoulos GK. Liverspecific ablation of integrin-linked kinase in mice results in abnormal histology, enhanced cell proliferation, and hepatomegaly. Hepatology 2008;48:1932-41.

5. Gkretsi V, Bowen WC, Yang Y, Wu C, Michalopoulos GK. Integrinlinked kinase is involved in matrix-induced hepatocyte differentiation. Biochem Biophys Res Commun 2007;353:638-43.

6. Kensler TW, Qian GS, Chen JG, Groopman JD. Translational strategies for cancer prevention in liver. Nat Rev Cancer 2003;3:321-9.

7. Jiang WG, Sanders AJ, Katoh M, Ungefroren H, Gieseler F, Prince M, Thompson SK, Zollo M, Spano D, Dhawan P, Sliva D, Subbarayan PR, Sarkar M, Honoki K, Fujii H, Georgakilas AG, Amedei A, Niccolai E, Amin A, Ashraf SS, Ye L, Helferich WG, Yang X, Boosani CS, Guha G, Ciriolo MR, Aquilano K, Chen S, Azmi AS, Keith WN, Bilsland A, Bhakta D, Halicka D, Nowsheen S, Pantano F, Santini D. Tissue 
invasion and metastasis: molecular, biological and clinical perspectives. Semin Cancer Biol 2015; pii: S1044-579X(15)00023-1. doi: 10.1016/j. semcancer.2015.03.008.

8. Canel M, Serrels A, Frame MC, Brunton VG. E-cadherin-integrin crosstalk in cancer invasion and metastasis. J Cell Sci 2013;126:393-401.

9. Chen L, Yang S, Jakoncic J, Zhang JJ, Huang XY. Migrastatin analogues target fascin to block tumour metastasis. Nature 2010;464:1062-6.

10. Huang X, Ji J, Xue H, Zhang F, Han X, Cai Y, Zhang J, Ji G. Fascin and cortactin expression is correlated with a poor prognosis in hepatocellular carcinoma. Eur J Gastroenterol Hepatol 2012;24:633-9.

11. Iguchi T, Aishima S, Umeda K, Sanefuji K, Fujita N, Sugimachi K, Gion T, Taketomi A, Maehara Y, Tsuneyoshi M. Fascin expression in progression and prognosis of hepatocellular carcinoma. J Surg Oncol 2009;100:575-9.

12. Gkretsi V, Bogdanos DP. Experimental evidence of Migfilin as a new therapeutic target of hepatocellular carcinoma metastasis. Exp Cell Res 2015;334:219-27.

13. Zhang Y, Tu Y, Gkretsi V, Wu C. Migfilin interacts with vasodilatorstimulated phosphoprotein (VASP) and regulates VASP localization to cell-matrix adhesions and migration. J Biol Chem 2006;281:12397-407.

14. Gkretsi V, Zhang Y, Tu Y, Chen K, Stolz DB, Yang Y, Watkins SC, Wu C. Physical and functional association of migfilin with cell-cell adhesions. $J$
Cell Sci 2005;118:697-710.

15. Tu Y, Wu S, Shi X, Chen K, Wu C. Migfilin and Mig-2 link focal adhesions to filamin and the actin cytoskeleton and function in cell shape modulation. Cell 2003;113:37-47.

16. Castellano F, Le Clainche C, Patin D, Carlier MF, Chavrier P. A WASpVASP complex regulates actin polymerization at the plasma membrane. EMBO J 2001;20:5603-14.

17. Sechi AS, Wehland J. ENA/VASP proteins: multifunctional regulators of actin cytoskeleton dynamics. Front Biosci 2004;9:1294-310.

18. Kwiatkowski AV, Gertler FB, Loureiro JJ. Function and regulation of Ena/VASP proteins. Trends Cell Biol 2003;13:386-92.

19. Adams JC. Fascin protrusions in cell interactions. Trends Cardiovasc Med 2004;14:221-6.

20. Tan VY, Lewis SJ, Adams JC, Martin RM. Association of Fascin-1 with mortality, disease progression and metastasis in carcinomas: a systematic review and meta-analysis. BMC Med 2013;11:52.

21. Kulasingam V, Diamandis EP. Fascin-1 is a novel biomarker of aggressiveness in some carcinomas. BMC Med 2013;11:53.

22. Hayashi Y, Osanai M, Lee GH. Fascin-1 expression correlates with repression of E-cadherin expression in hepatocellular carcinoma cells and augments their invasiveness in combination with matrix metalloproteinases. Cancer Sci 2011;102:1228-35. 